\title{
Ellipsometric Study of the Liquid/Liquid Interface in a Phase-Separated Colloid-Polymer Suspension
}

\author{
Els H. A. de Hoog, ${ }^{\dagger}$ Henk N. W. Lekkerkerker, ${ }^{* \dagger}{ }^{\dagger}$ Jens Schulz, ${ }^{\ddagger}$ and Gerhard H. Findenegg ${ }^{\ddagger}$ \\ Van 't Hoff Laboratory for Physical and Colloid Chemistry, Utrecht University, Padualaan 8 , \\ 3584 CH Utrecht, The Netherlands, and Iwan N. Stranski Institute of Physical and Theoretical Chemistry, \\ Technical University Berlin, Strasse des 17. Juni 112, D-10623 Berlin, Germany
}

Received: June 25, 1999; In Final Form: September 10, 1999

\begin{abstract}
An investigation of the interface in a phase-separated colloid-polymer suspension with ellipsometry is presented. The coefficient of ellipticity at the Brewster angle of light reflected at the interface between the coexisting colloid-rich and polymer-rich phase was measured along two trajectories through the phase diagram. Interpreting the data in terms of a diffuse interface model, an interfacial thickness in the order of the diameter of the colloidal particles is found.
\end{abstract}

\section{Introduction}

In the past few decades, phase separation in suspensions of colloidal particles and free, nonadsorbing polymers have been studied extensively. It is now generally believed that phase separation in these mixtures occurs due to the depletion interaction between the colloids, induced by the polymers. ${ }^{1,2}$ The depletion interaction can be understood as follows. Around the colloidal particles is a shell into which the mass centers of the polymers are unable to penetrate, the so-called depletion zones. When the colloidal particles approach each other close enough for the depletion zones to overlap, an imbalance in osmotic pressure is exerted by the polymers, which leads to an effective attraction between the colloids. The size of the polymers and colloids determines the range of the associated depletion potential, and the concentration of the polymers determines the depth of this potential; change in sizes or concentrations will have an influence on the depletion potential. Colloid-polymer suspensions phase-separate into a colloid-rich phase and a polymer-rich phase when the attraction is sufficiently strong. This transition can either be liquid-liquid or fluid-solid. The mechanism and kinetics of these phase transitions have been studied. ${ }^{3-6}$

The formation and the structure of the interface between the coexisting phases in a demixed colloid-polymer suspension are important factors in phase separation kinetics and in emulsification processes. Recently, we have measured the interfacial tension in such suspensions, ${ }^{7}$ which is in the range $0.0030-$ $0.0045 \mathrm{mN} / \mathrm{m}$, depending on the concentrations of the colloids and the polymers. This interfacial tension is very low in comparison with atomic gas-liquid interfaces $(\sim 10-100 \mathrm{mN} /$ $\mathrm{m}$ ) and also low in comparison with polymeric systems (a few $\mathrm{mN} / \mathrm{m})$. We observed a flat and sharp interface in these demixed suspensions. As far as we know, the structure of these kinds of interfaces has not been investigated before. The aim of this work is to gain some information concerning the thickness of the interfacial region of this phase-separated colloid-polymer suspension. Such information can be obtained by ellipsometry.

* Corresponding author. E-mail: H.N.W.Lekkerkerker@chem.uu.nl.

$\dagger$ Utrecht University.

$\doteqdot$ Technical University Berlin.
Ellipsometry ${ }^{8}$ is a well-established technique to determine the thickness of submicrometer films at surfaces and interfaces. It measures the state of polarization of light reflected at the interface, expressed by the ratio $r=r_{\mathrm{p}} / r_{\mathrm{s}}$, where $r_{\mathrm{p}}$ and $r_{\mathrm{s}}$ are the reflected amplitudes of light, polarized parallel and perpendicular to the plane of incidence. The coefficient of ellipticity $\bar{\rho}$ equals $\operatorname{Im}(r)$ at the Brewster angle $\Theta_{\mathrm{B}}$. When the interface is perfectly sharp and flat, $\bar{\rho}$ is zero. Deviations from this zero value can be attributed to a gradual change of the composition through the interface (diffuse interface) ${ }^{9}$ or to roughness caused by thermally excited capillary waves. ${ }^{10,11}$ Scattering of the incident or reflected light (as to be expected in the study of a colloidal suspension) should not change the phase of the light and thus should not affect the ellipticity.

This paper is organized as follows. In section 2, the colloidpolymer suspension, the ellipsometer, and the measurement procedure are described. Section 3 gives the results of the ellipsometric measurements. In section 4 , a discussion on the measurements and the results is given, followed by the conclusions in section 5 .

\section{Experiment}

System. We used mixtures of spherical silica colloids and poly(dimethylsiloxane) polymers in the solvent cyclohexane. This system was originally prepared and characterized by Verhaegh in a study of the liquid-liquid transition. ${ }^{12}$ The colloids are commercially available Ludox spheres (Ludox AS $40 \%$, Dupont) coated with 1-octadecanol (Merck, zur Synthese); the hydrodynamic radius of the particles is $13 \mathrm{~nm}$ with a polydispersity of $19 \%$, and the particle density is $1.60 \mathrm{~g} / \mathrm{mL}$. The polymer is commercially available poly(dimethylsiloxane) (PDMS, Janssen); the radius of gyration of the polymers was determined to be $14 \mathrm{~nm}$, its molecular weight $97000 \mathrm{~g} / \mathrm{mol}\left(M_{\mathrm{w}} /\right.$ $M_{\mathrm{n}}=1.9$ ), and the density $0.976 \mathrm{~g} / \mathrm{mL}$. The dispersions are almost transparent, because the refractive index of the particles nearly matches that of cyclohexane. However, the optical contrast is sufficiently good to allow clear observation of the interface between the coexisting liquid-liquid phases.

After homogenization of a colloid-polymer mixture at sufficiently high concentrations of colloids and polymers, the system first becomes turbid, and after a short time the turbidity 


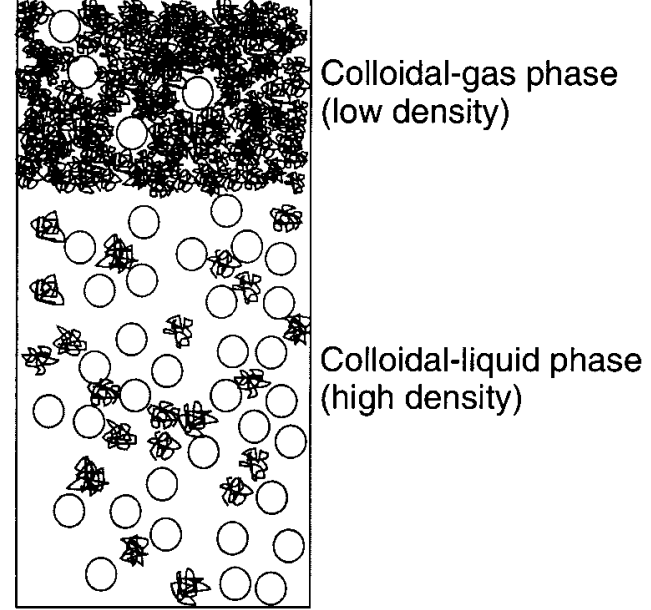

Figure 1. Artist's impression of a colloid-polymer suspension separated in a colloidal gas and a colloidal liquid phase.

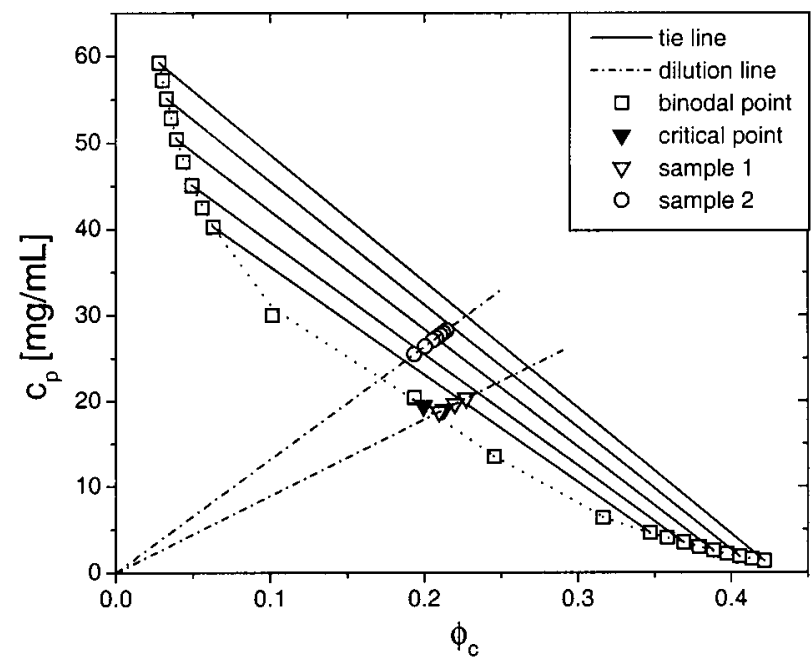

Figure 2. Constructed phase diagram of the colloid-polymer suspension where the polymer concentration, $c_{\mathrm{p}}$, is plotted vs the colloid volume fraction, $\phi_{c}$; in this diagram are shown the measured and a number of the calculated binodal ( $\square$ ), some of the calculated tie lines $(-)$, the critical point $(\boldsymbol{\nabla})$ estimated by extrapolating the midpoints of the tie lines to the binodal, the composition of measurements in sample $1(\nabla)$ and in sample $2(O)$, and the dilution lines of the samples $(---)$.

reduces and the formation of an interface is observed. The system phase-separates into a colloid-rich (colloidal liquid) and a polymer-rich (colloidal gas) phase; see Figure 1. Phase separation typically takes place within half an hour to a few hours, depending on the concentrations. With a method proposed by Bodnár and Oosterbaan, ${ }^{13}$ the boundary between the oneand two-phase regions and the tie lines in the phase diagram were determined, as described elsewhere. ${ }^{7}$ This method is based only on volume measurements of the coexisting phases along at least three dilution lines (i.e., straight lines through the phase diagram with a constant colloid-to-polymer concentration ratio) and on the mass balance. The phase diagram is shown in Figure 2. It gives the measured binodal points, some of the calculated binodal points, some of the calculated tie lines, the critical point, the composition of the samples used in the experiments, and the dilution lines of the samples. The binodal points are connected with a dotted line to guide the eye. The critical point was estimated by extrapolating the midpoints of the tie lines to the binodal. The compositions of coexisting phases are determined from the phase diagram as follows. The overall concen- trations of the colloids and polymers in a suspension indicate the position in the phase diagram. The tie line through this point in the phase diagram determines the points on the binodal of the two coexisting phases and therefore the concentrations of the colloids and the polymers in both phases. The density of both phases can then be calculated with the known densities of the colloids, the polymers, and the solvent.

Ellipsometer. A phase modulation ellipsometer was used to measure the coefficient of ellipticity of light reflected at the interface. ${ }^{14}$ The optical configuration consists of a $10 \mathrm{~mW} \mathrm{He}-$ Ne laser $(\lambda=632.8 \mathrm{~nm})$, a polarizer (Glan-Thompson-Prism), a phase modulator (Hinds, model FS5), the sample cell, an analyzer, and a photomultiplier tube as the detector. The sample cell was made of a cylindrical glass tube and was held in place by a support such that the axis of the cylinder was perpendicular to the plane of incidence of the light beam. The cell had two outlets: one was used to add solvent and was closed with a Teflon tap; in the second outlet, a Teflon stick was inserted. By changing the position of this stick in the outlet it was possible to adjust the level of the interface in the sample. In all measurements, it was attempted to keep the level of the interface exactly in the axial plane of the cylindrical cell.

Measurements. Ellipticity measurements were made on two samples with different colloid-to-polymer concentration ratios, as indicated in Figure 2. The concentrations in both samples were varied by dilution with the solvent; with this procedure, the colloid-to-polymer concentration ratio remains constant. The incident light passes through the upper, polymer-rich phase at the Brewster angle, which was found to be $44.7^{\circ}$ for a suspension with a colloid volume fraction of 0.222 and a polymer concentration of $21.5 \mathrm{mg} / \mathrm{mL}$.

The experiment was conducted as follows. A small amount of solvent was added to the sample; portions were varied from 0.025 to $0.5 \mathrm{~mL}$. The sample holder with the sample cell was taken out of the apparatus and slowly shaken until the sample was homogeneously turbid. The sample holder was replaced carefully into the same position in the apparatus. After a few hours, phase separation was completed and the ellipticity was measured. This procedure was repeated three times for sample 1 and 10 times for sample 2 .

\section{Results}

The ellipticity was measured as a function of the difference in density between the coexisting phases along dilution lines. Two samples, indicated in Figure 2, with different colloid-topolymer concentration ratios were studied. The results of the measurements at 4 state points in sample 1 and at 11 state points in sample 2 are given in Tables 1 and 2. In both cases, no systematic variation of $\bar{\rho}$ as a function of the sample composition was found. The average value of the measured ellipticity is +0.0041 in sample 1 and -0.0081 in sample 2. In Figure 3, the measured ellipticity is given as a function of the difference in density of the coexisting phases, $d_{\alpha}-d_{\beta}$, which indicates the distance to the critical point. The density difference of sample 1 at state points $1-3$ was estimated by extrapolation of the tie lines.

TABLE 1: Measurement Ellipticity of Sample 1

\begin{tabular}{cccc}
\hline & $\begin{array}{c}c_{\mathrm{p}} \\
{[\mathrm{mg} / \mathrm{mL}]}\end{array}$ & $\begin{array}{c}d_{\alpha}-d_{\beta} \\
{[\mathrm{mg} / \mathrm{mL}]}\end{array}$ & $\rho_{\mathrm{m}} \times 10^{-3}$ \\
\hline 0.210 & 18.7 & 153 & 4.5 \\
0.213 & 19.0 & 179 & 4.4 \\
0.220 & 19.7 & 213 & 3.1 \\
0.227 & 20.3 & 237 & 4.4
\end{tabular}


TABLE 2: Measurement Ellipticity of Sample 2

\begin{tabular}{cccc}
\hline \multicolumn{1}{c}{$c_{\mathrm{p}}$} & $\begin{array}{c}d_{\alpha}-d_{\beta} \\
{[\mathrm{mg} / \mathrm{mL}]}\end{array}$ & $\bar{\rho}_{\mathrm{m}} \times 10^{-3}$ \\
\hline 0.1935 & 25.5 & 248 & -8.5 \\
0.2004 & 26.4 & 264 & -8.6 \\
0.2053 & 27.1 & 274 & -9.2 \\
0.2078 & 27.4 & 279 & -8.2 \\
0.2091 & 27.6 & 282 & -6.9 \\
0.2104 & 27.7 & 284 & -7.0 \\
0.2118 & 27.9 & 286 & -7.4 \\
0.2124 & 28.0 & 287 & -8.3 \\
0.2131 & 28.1 & 289 & -7.6 \\
0.2138 & 28.2 & 290 & -8.7 \\
0.2144 & 28.3 & 291 & -8.5
\end{tabular}

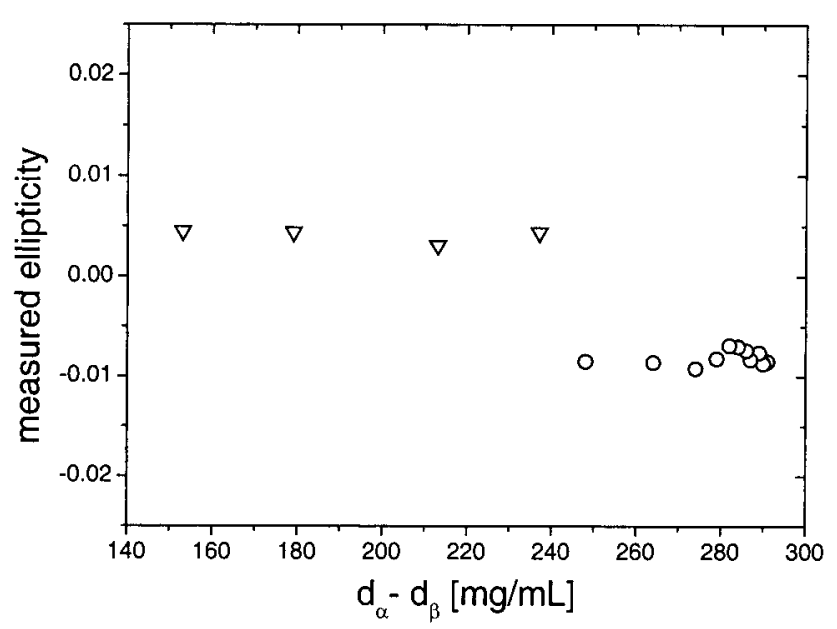

Figure 3. Measured ellipticity as a function of the difference in density, $d_{\alpha}-d_{\beta}$, between the coexisting phases along two dilution lines: $(\nabla)$ sample $1 ;(\bigcirc)$ sample 2.

A significant contribution to the measured ellipticity comes from the birefringence of the glass walls of the sample cell, and the magnitude of this contribution depends on precise positions at which the laser beam is passing through the glass wall. Care was taken to keep the sample cell in precisely the same position during the measurements of the dilution lines. The fact that the measured ellipticities are positive for sample 1 and negative for sample 2 may indeed be attributed to the spurious birefringence effect of the glass. For sample 1, we determined the contribution to $\bar{\rho}$ due to the glass cell by reference measurements of the liquid/liquid interface of the butanol + water system for which the ellipticity is known. ${ }^{10} \mathrm{In}$ this way, the contribution due to birefringence was found to be +0.0036 for sample 1. Accordingly, the net ellipticity of the interface in sample 1 is +0.0005 .

The ellipticity was analyzed in terms of the diffuse interface model. Assuming that the composition profile of the interface can be expressed in terms of a refractive index profile $n(z)$ between two isotropic phases $\alpha$ and $\beta$, the ellipticity $\bar{\rho}_{i}$ of the interface is given by the approximate Drude equation: ${ }^{15}$

$\bar{\rho}_{i}=\frac{\pi\left(n_{\alpha}{ }^{2}+n_{\beta}{ }^{2}\right)^{1 / 2}}{\lambda\left(n_{\alpha}{ }^{2}-n_{\beta}{ }^{2}\right)} \int_{-\infty}^{+\infty} \frac{\left(n_{\alpha}{ }^{2}-n(z)^{2}\right)\left(n(z)^{2}-n_{\beta}{ }^{2}\right)}{n(z)^{2}} \mathrm{~d} z$

Here, $\lambda$ is the wavelength of the incident light and $n_{\alpha}$ and $n_{\beta}$ are the refractive indices of the two phases. In the present experiment, phase $\beta$ (defined as the phase through which the light is transmitted to the interface) was the polymer-rich (low density) phase, which had a lower refractive index than the colloid-rich phase $\alpha$ (i.e., $n_{\alpha}-n_{\beta}>0$ ). Near the critical point, the interfacial profile is expected to be symmetric and can be written in the form ${ }^{16,17}$

$$
n(z)=\frac{1}{2}\left(n_{\alpha}+n_{\beta}\right)-\frac{1}{2}\left(n_{\alpha}-n_{\beta}\right) X
$$

with $X(+\infty)=+1$ and $X(-\infty)=-1$.

In the present analysis, the mean-field profile function $X_{\mathrm{MF}}$ $=\tanh (z / 2 \xi)$ was adopted, where $\xi$ is a measure for the thickness of the interface. In the van der Waals-Cahn-Hilliard theory, this length, $\xi$, is identical with the correlation length of the critical fluctuations in the coexisting bulk phases. ${ }^{18}$ Since the refractive index differences between the two phases are very small for the present samples, $n_{\alpha}-n_{\beta} \ll n_{\alpha}, n_{\beta}$ and $n_{\alpha} \approx n_{\beta} \approx$ $n$, one obtains

$$
\bar{\rho}_{i}=2 \pi \sqrt{2}\left(n_{\alpha}-n_{\beta}\right) \xi / \lambda
$$

where the relation for the mean-field profile function

$$
\int_{-\infty}^{+\infty}\left[1-X_{\mathrm{MF}}^{2}(z / 2 \xi)\right] \mathrm{d}(z / 2 \xi)=2
$$

was used.

Equation 3 was used to determine $\xi$ for the present system. With the experimental data of sample 1 at state point $4\left(\phi_{\mathrm{c}}=\right.$ 0.227 and $\left.c_{\mathrm{p}}=20.3 \mathrm{mg} / \mathrm{mL}\right)$, i.e., $\bar{\rho}_{i}=0.0005, n_{\alpha}=1.4385$, $n_{\beta}=1.4250$, and $\lambda=632.8 \mathrm{~nm}$, we find $\xi=2.6 \mathrm{~nm}$. The thickness of the diffuse interfacial region may be expressed by the " $10-90$ " thickness $t,{ }^{19}$ for which eq 2 gives $t \approx 4.4 \xi$, i.e., ca. $11 \mathrm{~nm}$. A similar analysis of the data for sample 2 is not possible, because the birefringence contribution due to the sample cell is not known.

\section{Discussion}

The measurements reported above were limited by several experimental problems. The main obstacle was the large contribution to the ellipticity resulting from the birefringence of the glass cell. Whereas in studies of the temperature dependence of the ellipticity ${ }^{15}$ the sample cell is kept in position during a full temperature scan (and thus any birefringence effects remain constant), in the present study the sample cell had to be removed from the ellipsometer for each new experimental point along the dilution line, to guarantee thorough mixing of the sample. A related uncertainty arises from the difficulty in keeping the interface at precisely the same level in the sample cell before and after dilution. In practice, this turned out to be difficult to handle, although the design of the sample cell allowed for such adjustments of the interface. The nonsystematic scatter of the measured ellipticities along the dilution lines of the two samples (Tables 1 and 2) are believed to be caused mainly by spurious birefringence effects due to the imprecise repositioning of the sample cell and interface level.

A systematic error of the ellipticities of sample 1 can arise from errors in the reference measurements on the butanol + water system. Such errors are estimated to be less than 0.0005 in $\bar{\rho}$. Furthermore, the Brewster angle (which depends on the refractive indices of the two phases) was determined only for one sample, although the refractive indices will vary along the dilution line.

As mentioned before, the birefringence contribution to the ellipticities was determined only for sample 1, and thus the magnitude of the ellipticity of the interface in sample 2 could not be determined. Nevertheless, it can be stated that in both samples the ellipticity does not exhibit a significant dependence on the composition of the sample along the dilution line 
(expressed as the density difference between the coexisting phases) in the phase diagram. We may discuss this finding in terms of eq 3 . Inspection of Figure 2 shows that the refractive increment, $n_{\alpha}-n_{\beta}$, decreases with decreasing density difference along the experimental paths of sample 1 and 2 . The observation that the ellipticity of the interface remains constant along these trajectories then implies an increase of the interfacial thickness as $\xi \propto\left(n_{\alpha}-n_{\beta}\right)^{-1}$. As the critical point is approached, the compositions of the coexisting phases and the refractive index increment, $n_{\alpha}-n_{\beta}$, will converge to zero while the correlation length, $\xi$, will increase and diverges as one approaches the critical point. The increase of $\xi$ will dominate the behavior of the ellipticity sufficiently close to the criticality of the system. However, further away from the critical point, the two antagonistic effects of $n_{\alpha}-n_{\beta}$ and $\xi$ may result in a nearly constant value of the ellipticity. Indeed, in previous studies of near-critical interfaces in simple two-component systems (where the ellipticity was measured as a function of the distance from the critical temperature $\left|T_{\mathrm{c}}-T\right|$ ), it was found ${ }^{14,15}$ that the ellipticity exhibits only a weak temperature dependence down to $\left|T_{\mathrm{c}}-T\right|$ $\approx 3 \mathrm{~K}$ (corresponding to a relative distance $\left|T_{\mathrm{c}}-T\right| / T_{\mathrm{c}} \approx 10^{-2}$ ). Thus, it is likely that a significant increase in the magnitude of the ellipticity will occur only very close to the critical point. To test this conjecture, it would be necessary to locate the critical point more accurately than has been possible so far.

In the present analysis it has been presumed that the behavior of the interface of this three-component system can be analyzed in terms of a single density variable. In principle, the concentration profile of the colloid and of the polymer might vary in a different manner, e.g., the concentration profile of the colloid particles might be a monotonic function of the coordinate $z$ while the profile of the polymer might exhibit a maximum or minimum in the interface. In such a case the refractive index profile $n(z)$ may deviate from a simple symmetric form as in eq 2 . Specifically, if $n(z)$ exhibits a maximum in the interfacial region this would cause a negative contribution to the integral in eq 1 . In this case, the value $\bar{\rho}_{i}$ observed for sample $1(+0.0005)$ would imply a greater thickness than the estimated $10-90$ value $t=$ $11 \mathrm{~nm}$. Further studies are necessary to investigate this possibility.

\section{Conclusion}

We have shown that ellipsometry can be used to study the thickness of the interface of a phase-separated colloid-polymer suspension. It must be emphasized that the measurements reported in this work are preliminary. We found that the interface can be considered in terms of the diffuse interface model, with gradual changes in the colloid and polymer concentration from one phase to the other. No significant change of the ellipticity along the chosen trajectories in the two-phase region was found, although the refractive index difference, $n_{\alpha}-n_{\beta}$, between the coexisting phases changes along this path. This finding implies that the interfacial thickness increases as one approaches the critical point. On the other hand, the composition region studied in this work appears to be too far away from the critical point in order to detect an increase in ellipticity, which is a signature of critical interfaces. A rough estimate of the thickness of the interfacial region yields a value on the order of $10 \mathrm{~nm}$, which is not inconsistent with the expected value of the diameter of the colloid particles.

Acknowledgment. We thank Peter de Graaf and Kees Rietveld for their technical support. This work was supported by the Stichting voor Fundamenteel Onderzoek der Materie (Foundation for Fundamental Research on Matter) which is part of the Nederlandse Organisatie voor Wetenschappelijk Onderzoek (Netherlands Organization for the Advancement of Research) and by the Deutsche Forschungsgemeinschaft (DFG) under Grant FI 235/11-3.

\section{References and Notes}

(1) Asakura, S.; Oosawa, F. J. Chem. Phys. 1954, 22, 1255

(2) Vrij, A. Pure Appl. Chem. 1976, 48, 471.

(3) Cowell, C.; Lin-In-On, R.; Vincent, B. J. Chem. Soc., Faraday Trans. 1 1978, 74, 337

(4) Gast, A. P.; Hall, C. K.; Russel, W. B. J. Colloid Interface Sci. 1983, 96, 251.

(5) Lekkerkerker, H. N. W.; Poon, W. C. K.; Pusey, P. N.; Stroobants, A.; Warren, P. B. Europhys. Lett. 1992, 20, 559.

(6) Ilett, S. M.; Orrock, A.; Poon, W. C. K.; Pusey, P. N. Phys. Rev. E 1995, 51, 1344

(7) de Hoog, E. H. A.; Lekkerkerker, H. N. W. J. Phys. Chem. B 1999, 103,5274

(8) Azzam, R. M.; Bashara, N. M. Ellipsometry and Polarized Light; North-Holland Publishing Co.: Amsterdam, 1977.

(9) Drude, P. The Theory of Optics; Dover: New York, 1959.

(10) Meunier, J. J. Phys. (Paris) 1987, 48, 1819.

(11) Zielinska, B. J. A.; Bedeaux, D.; Vlieger, J. Physica A 1981, 10 791.

(12) Verhaegh, N. A. M.; van Duijneveldt, J. S.; Dhont, J. K. G.; Lekkerkerker, H. N. W. Physica A 1996, 230, 409.

(13) Bodnár, I.; Oosterbaan, W. D. J. Chem. Phys. 1997, 106, 7777.

(14) Heidel, B.; Findenegg, G. H. J. Chem. Phys. 1987, 87, 706.

(15) Schulz, J.; Hirtz, A.; Findenegg, G. H. Physica A 1997, 244, 334.

(16) Fisk, S.; Widom, B. J. Chem. Phys. 1969, 50, 3219.

(17) Schmidt, J. W. Physica A 1991, 172, 40.

(18) Rowlinson, J. S.; Widom, B. Molecular Theory of Capillarity; Clarendon Press: Oxford, 1982.

(19) Beaglehole, B. Physica B 1982, 112, 320 